\title{
Performance of converted pressure cookers and two conventional jars for anaerobic bacterial culture
}

\author{
R. A. GARGAN ${ }^{1}$ AND I. PHILlips \\ From the Department of Microbiology, St. Thomas's Hospital Medical School, London SE1 7EH, UK
}

SUMMARY The simple conversion of commercial pressure cookers into inexpensive anaerobic jars is described. These containers were shown to be as good as the small conventional BBL poly-? carbonate GasPak and large vented 150 gas-replacement jars when assessed by means of three $\stackrel{\omega}{-}$ biological indicators: Pseudomonas aeruginosa, Bacteroides melaninogenicus, and Bacteroides or fragilis. Ps. aeruginosa seeded on Simmond's citrate agar was shown to be the most sensitive $\stackrel{\vec{O}}{\circ}$ indicator of the three for traces of oxygen.

A variety of anaerobic jars is at present available commercially, varying in construction from the well-known Baird and Tatlock steel anaerobic jar to the transparent polycarbonate BBL GasPak and gas-replacement designs. Most of these anaerobic jars are fairly expensive, and so we decided to evaluate an alternative, less expensive piece of apparatus-a commercial pressure cooker with minor modifications to make it suitable as an anaerobic jar. The performance of this experimental anaerobic jar was compared with that of two types of polycarbonate anaerobic jars marketed by Becton Dickinson (UK) Ltd: the large vented BBL 150 jars with a gas-replacement system, and the small BBL GasPak jars with the GasPak system.

A biological indicator system consisting of three organisms was chosen for the assessment of the anaerobic jars. The first was Pseudomonas aeruginosa, which does not grow in the absence of oxygen but will grow if only small amounts are present. Collee (personal communication) suggested that cultures on nutrient agar or Blood Agar Base No. 2 (Oxoid) might be better than chemical indicators in detecting small amounts of oxygen in anaerobic jars. However, we found that pseudomonas did grow on these media under anaerobic conditions, albeit poorly, presumably by utilising alternatives to oxygen in electron transport. Simmond's citrate agar (Oxoid) supports the luxuriant growth of pseudomonas in air but does not contain these oxygen alternatives. Furthermore, even slight

${ }^{1}$ Present address: Department of Microbiology, Royal Free Hospital, Pond Street, London NW3

Received for publication 7 October 1977 growth is detectable by the change from green to blue of the bromo-thymol blue indicator. It therefore seemed a suitable medium on which to inoculate Ps. aeruginosa as an oxygen detector. The two. other organisms chosen as part of the indicator system were Bacteroides melaninogenicus subspecies asaccharolyticus and Bacteroides fragilis ss fragilis, highly and moderately strict, respectively, in their requirement for anaerobic conditions $\subseteq$ (Loesche, 1969; Tally et al., 1975). We have $\vec{\circ}$ assumed that extremely oxygen-sensitive organisms 3 are not important in human clinical material (Rosenblatt et al., 1973).

\section{Material and methods}

DESCRIPTION OF ANAEROBIC JARS

Two large 8-litre Tefal aluminium pressure cookers, internal diameter $24 \mathrm{~cm}$, internal height $17 \mathrm{~cm}$, capable of holding 40 standard plastic Petri dishes (Figure, left jar) and two smaller 6-litre Tefalo stainless steel pressure cookers, internal diameter $22 \mathrm{~cm}$, internal height $15 \mathrm{~cm}$, holding up to $30 \mathrm{~N}$ Petri dishes (Figure, right jar), were purchased $N$ from the importers (Liberta Imex, 13 Consul Road, N Rugby CV21 1PB, UK). Both sizes of pressure cooker were available in aluminium and stainless steel.

The conversion into an anaerobic container was made by discarding the weighted steam pressure regulator and attaching a short piece of surgical ${ }^{-}$ rubber tubing with a clamp to the steam outlet nozzle. The safety valve was then replaced with $a \frac{\text { ? }}{\mathbb{D}}$ tight-fitting rubber bung. A catalyst sachet of woven $\frac{}{2}$ wire 80 mesh, 39 gauge stainless steel (Greenwich? Metal Works, Woolwich Road, London SE7), 426 


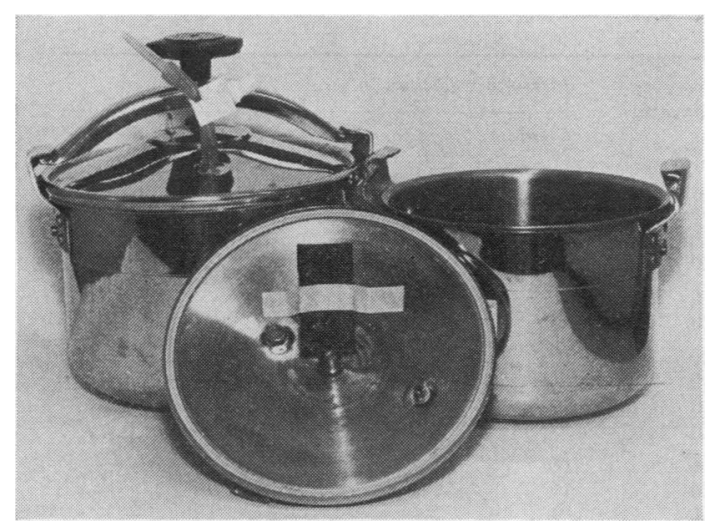

Figure Two pressure cookers converted for use as anaerobic jars.

containing approximately $10 \mathrm{~g}$ of room-temperature palladium catalyst pellets (Becton Dickinson (UK) Ltd) was secured to the inside of the lid with a strip of adhesive tape ${ }^{1}$. Three standard catalyst sachets, as used in Baird and Tatlock anaerobic jars, secured in the same manner were also found to be suitable.

The polycarbonate anaerobic jars were of two different types: large vented BBL 150 jars (Becton Dickinson) and small unvented BBL GasPak jars (Becton Dickinson). Four large vented BBL 150 jars were used with the gas replacement system and four small BBL GasPak jars with the GasPak anaerobic system.

The pressure cookers and vented BBL 150 jars were purchased new at the beginning of this study. The small GasPak jars were already in general use but appeared to be functioning well.

\section{BIOLOGICAL INDICATORS}

For each investigation, Ps. aeruginosa NCTC 10662 was inoculated on Simmons' citrate agar (Oxoid, CM155) in two Petri dishes, one for anaerobic incubation and the other for aerobic incubation as a control of growth and as a source of pseudomonas in subsequent studies. Both strains of bacteroides, which were recent clinical isolates, were grown in Robertson's cooked meat broth and subcultured every five days, and a loopful of each broth was used to inoculate the respective media. The strain of B. melaninogenicus ss asaccharolyticus was inoculated on Columbia (Oxoid, CM331) lysed blood agar (Finegold et al., 1971) and the strain of $B$. fragilis ss fragilis on Columbia whole blood agar.

${ }^{1}$ We later used a metal strip, bolted to the under surface of one of the outlets, as a means of securing the sachet.
ANAEROBIC PROCEDURE

Citrate, lysed- or whole-blood-agar plates inoculated with the three different organisms were incubated in each of the 12 anaerobic jars for 48 hours at $37^{\circ} \mathrm{C}$, and the results of growth were recorded after each incubation period. The converted pressure cookers and the vented BBL 150 jars were evacuated to $-600 \mathrm{~mm}$ of mercury with a vacuum pump and filled with a gas mixture comprising $10 \%$ hydrogen, $10 \%$ carbon dioxide, and $80 \%$ nitrogen (British Oxygen Co Ltd). The BBL GasPak jar received a disposable GasPak aluminium foil envelope (Becton Dickinson (UK) Ltd) which was activated with $10 \mathrm{ml}$ of distilled water. Catalyst sachets were removed from the lids of the experimental jars, and the catalyst holders of the BBL jars were emptied after each usage. All catalyst was reactivated by heating to $160^{\circ} \mathrm{C}$ for two hours in a hot air oven (Brewer and Allgeier, 1966).

Three extra Tefal pressure cookers-one large 8-litre stainless steel jar and two small 6-litre stainless steel jars-were purchased and converted into anaerobic jars. All seven modified cookers were then put into routine use in our diagnostic laboratory with Ps. aeruginosa inoculated on Simmons' citrate agar as the sole anaerobic indicator in each jar.

\section{Results}

SENSITIVITY OF THE BIOLOGICAL

INDICATOR SYSTEM

Ps. aeruginosa was found to be a more sensitive indicator of the presence of traces of oxygen than the strain of $B$. melaninogenicus and much more sensitive than $B$. fragilis. The various states of anaerobiosis in the jars were designated good, moderate, poor, or aerobic. Good anaerobic conditions prevented the growth of Ps. aeruginosa while permitting the growth of both $B$. melaninogenicus and $B$. fragilis. A moderate degree of anaerobiosis allowed the growth of Ps. aeruginosa while still permitting the growth of $B$. melaninogenicus and $B$. fragilis. Poor anaerobic conditions permitted the growth of $P s$. aeruginosa and $B$. fragilis while inhibiting the growth of $B$. melaninogenicus.

\section{PERFORMANCE OF THE THREE ANAEROBIC JARS}

Three of the four vented BBL 150 jars presented a particular problem with malfunctioning lids due to the weakness of the plastic rims retaining the rubber sealing ring. These jars were tested 10,11 , and 16 times respectively before crccking of the rim became 
Table Performance of three types of anaerobic jars

\begin{tabular}{|c|c|c|c|c|c|c|}
\hline \multirow[t]{3}{*}{ Anaerobic jar type } & \multirow[t]{3}{*}{ Number } & \multirow{3}{*}{$\begin{array}{l}\text { Number } \\
\text { of tests }\end{array}$} & \multicolumn{4}{|c|}{ Inferred conditions in jar } \\
\hline & & & \multirow[t]{2}{*}{ Good } & \multicolumn{2}{|l|}{ Anaerobic } & \multirow[t]{2}{*}{ Aerobic } \\
\hline & & & & Moderate & Poor & \\
\hline Tefal 8-litre aluminium cooker & $\begin{array}{l}1 \\
2\end{array}$ & $\begin{array}{l}20 \\
20\end{array}$ & $\begin{array}{l}20 \\
17\end{array}$ & - & $\overline{3}$ & - \\
\hline Tefal 6-litre stainless steel cooker & $\begin{array}{l}1 \\
2\end{array}$ & $\begin{array}{l}20 \\
20\end{array}$ & $\begin{array}{l}20 \\
20\end{array}$ & - & 二 & - \\
\hline BBL GasPak jar & $\begin{array}{l}1 \\
2 \\
3 \\
4\end{array}$ & $\begin{array}{l}20 \\
10 \\
20 \\
20\end{array}$ & $\begin{array}{r}20 \\
4 \\
20 \\
17\end{array}$ & $\frac{-}{2}$ & $\begin{array}{l}\overline{4} \\
-\end{array}$ & $\frac{-\frac{2}{1}}{\frac{1}{1}}$ \\
\hline Vented BBL 150 jar & $\begin{array}{l}1 \\
2 \\
3 \\
4\end{array}$ & $\begin{array}{l}16 \\
20 \\
11 \\
10\end{array}$ & $\begin{array}{c}16 \\
19 \\
9 \\
1\end{array}$ & $\frac{\overline{1}}{1}$ & $\begin{array}{c}\overline{-} \\
2 \\
7\end{array}$ & $\frac{-}{1}$ \\
\hline
\end{tabular}

apparent, and they were then excluded from the study. An attempt has since been made by the manufacturer to rectify this fault.

The results of the performance of all the anaerobic jars are recorded in the Table. Three converted pressure cookers (one large 8-litre aluminium and two small 6-litre stainless steel cookers) performed well, giving good results in all 20 tests performed on them. One of the large 8-litre aluminium cookers, however, gave poor results on three occasions during 20 tests. Two BBL GasPak jars gave good results for the full 20 tests, one jar gave good results 17 times, and moderate results twice, and failed completely with aerobic conditions once. The final GasPak jar was excluded from the survey after 10 tests owing to a consistently unreliable performance, although no structural fault was apparent.

The first of the vented BBL 150 jars gave good results on 16 occasions before testing was abandoned owing to lid failure. The second jar gave good results 19 times and a moderate result once. The third gave good results nine times and poor results twice out of a total of 11 tests before lid failure. The last jar performed very poorly with good results once, moderate results once, poor results seven times, and complete failure once before the testing was discontinued owing to lid failure.

\section{PERFORMANCE OF MODIFIED PRESSURE COOKERS IN ROUTINE USE}

The seven containers, including the 8-litre aluminium container which originally gave poor results on three occasions during the study, continued to perform well, giving good results in constant daily use for nine months. After this period, however, failures in the anaerobic conditions of some of the jars began to be recorded. The rubber sealing rings were found to be at fault and, as a precaution, standard replacement rings (Tefal) were fitted to the lids? of all containers. These seven containers then worked successfully with no recorded growth of the pseudomonas on the citrate plates for a further year.

The jars have also been used for the isolation ${ }^{\infty}$ and identification of strict anaerobes from clinicalo material transported directly to the laboratory in oxygen-free, carbon-dioxide bottles. More than 500 strict anaerobes belonging to a wide range of genera, from deep wound infections, empyemas, $\stackrel{\mathbb{Q}}{\mathscr{\Omega}}$ abscesses, and closed septic lesions of the abdomen $\vec{\Rightarrow}$ associated with the gut or female genital tract, have 3 been isolated in them.

It might be thought that the catalyst sacheto would become too hot to be held in position by autoclave tape. In practice this has not been ao problem; on no occasion has it become detached $\frac{0}{3}$ or burned during use. However, the metal strip might be thought a safer means of attaching the sachet.

\section{Discussion}

Ps. aeruginosa on Simmons' citrate agar proved N to be the most sensitive indicator of the presenceof oxygen, as judged by its total lack of growth 0 in jars that were as anaerobic as possible. The fact $\omega$ that some jars permitted the growth of $B$. melaninogenicus while still allowing the growth of Ps.응 aeruginosa indicated that, while these jars worked well, they were not totally free of oxygen. The growth $\stackrel{?}{+}$ of $B$. fragilis became an indicator of a third, poor level of anaerobic conditions in jars where $P s . \stackrel{+}{\mathbb{D}}$ aeruginosa did grow but $B$. melaninogenicus did not. $\stackrel{\bigcirc}{\mathscr{Q}}$

It is arguable that the growth of a strict anaerobe $\stackrel{\unrhd}{\unrhd}$ such as Clostridium oedematiens type D (Watt et al., 1973) might have been a more exacting 8 
indicator of anaerobic conditions. However, the disadvantage of the difficulty of producing medium for a nutritionally fastidious organism would offset any advantage it might have as an indicator. The difficulty in this study of maintaining a pure and viable culture of $B$. melaninogenicus and of obtaining a consistently good growth on a solid medium suggests that this, too, is unsuitable as a routine indicator.

In this study we observed a wide variation in the performance of some of the anaerobic jars. Differences observed in the performance of the same Baird and Tatlock jar from day to day have been described by Watt et al. (1973) and called the "jar variation phenomenon'. These workers discussed possible explanations for the phenomenon, such as bacteriostatic substances in the anaerobic atmosphere and the effect of faulty catalyst. The first explanation could not be demonstrated by these workers with any consistency. We used freshly reactivated catalyst on every occasion in an attempt to avoid the second problem. It is possible that failures to achieve good anaerobic conditions in two of the BBL GasPak jars were due to occasional faulty GasPak hydrogen-carbon dioxide generators but this does not seem likely following the results of Collee et al. (1972), who found the GasPak anaerobic system reliable and consistent. Nor does it explain the large number of failures of one particular GasPak jar.

The large vented BBL 150 jars proved particularly troublesome with their faulty lids, and it was difficult therefore, to make an accurate assessment of their performance. However, the one jar which did not have a lid fault performed well, giving a moderate result on only one occasion in 20 tests.

Only five of the 12 jars tested gave consistently good results for 20 consecutive tests; these were three converted pressure cookers (one large 8-litre and two smaller 6-litre) and two BBL GasPak jars.

The most important feature of an anaerobic jar is its reliability in achieving anaerobic conditions. Our converted pressure cookers were as good in this respect as other jars specifically designed for the purpose and proved efficient in the isolation of a wide variety of strict anaerobes from clinical material over a period of one year and nine months. There appeared to be no significant difference between the construction of the aluminium and stainless steel containers which might affect anaerobic conditions.

The failure of the sealing rings in the lids of the containers after nine months occurred despite the fact that they were made of butyl rubber. The necessity of changing a sealing ring every nine months is not, however, a serious drawback.

The 8-litre and 6-litre containers were found to have a convenient capacity of 40 plates and 30 plates, respectively. The larger container was also found to be suitable for holding small racks of bottles. An added advantage was that API anaerobe microtest strips (Analytical Products Incorporated, Philpot House, Rayleigh, Essex) conveniently fitted into the large size container without any distortion or the need to stand the anaerobic jar on its side. The final important feature was the cost: a pressure cooker could be purchased and converted for about a quarter of the price of the average anaerobic jar. Furthermore, conversion of the pressure cooker into an anaerobic container is a simple process done in minutes with readily available material.

We thank Miss E. Taylor for her help in the longterm assessment of the anaerobic jars and $\mathrm{Mr} \mathrm{D}$. Davies for the figure.

\section{References}

Brewer, J. H., and Allgeier, D. L. (1966). Safe self contained carbon dioxide-hydrogen anaerobic system. Applied Microbiology, 14, 985-988.

Collee, J. G., Watt, B., Fowler, E. B., and Brown R. (1972). An evaluation of the GasPak system in the culture of anaerobic bacteria. Journal of Applied Bacteriology, 35, 71-82.

Finegold, S. M., Sugihara, P. T., and Sutter, V. L. (1971). Use of selective media for isolation of anaerobes from humans. In Isolation of Anaerobes, edited by D. A. Shapton and R. G. Board. Academic Press, London and New York.

Loesche, W. J. (1969). Oxygen sensitivity of various anaerobic bacteria. Applied Microbiology, 18, 723-727.

Rosenblatt, J. E., Fallon, A., and Finegold, S. M. (1973). Comparison of methods for isolation of anaerobic bacteria from clinical specimens. Applied Microbiology, 25, 77-85.

Tally, F. P., Stewart, P. R., Sutter, V. L., and Rosenblatt, J. E. (1975). Oxygen tolerance of fresh clinical anaerobic bacteria. Journal of Clinical Microbiology, 1, 161-164.

Watt, B., Hoare, M. V., and Collee, J. G. (1973). Some variables affecting the recovery of anaerobic bacteria: a quantitative study. Journal of General Microbiology, 77, 447-454.

Requests for reprints to: Professor I. Phillips, Department of Microbiology, St. Thomas' Hospital, London SE1 7EH 